\title{
Magnetization Reversal and Specific Loss Power of Magnetic Nanoparticles in Cellular Environment Evaluated by AC Hysteresis Measurement
}

\author{
Satoshi Ota, Tsutomu Yamada, and Yasushi Takemura \\ Department of Electrical and Computer Engineering, Yokohama National University, Yokohama 240-8501, Japan \\ Correspondence should be addressed to Yasushi Takemura; takemura@ynu.ac.jp
}

Received 30 January 2015; Accepted 5 May 2015

Academic Editor: Yong Zhu

Copyright (C) 2015 Satoshi Ota et al. This is an open access article distributed under the Creative Commons Attribution License, which permits unrestricted use, distribution, and reproduction in any medium, provided the original work is properly cited.

The effect of intracellular hyperthermia induced by magnetic nanoparticles (MNPs) has been evaluated using a theoretical model. In this study, magnetization reversal of MNPs in the cellular environment under an AC magnetic field was evaluated on the basis of measured AC hysteresis loops. The specific and intrinsic loss powers-SLP and ILP-were also estimated from the area of AC hysteresis loops. The measured samples were a liquid sample dispersed in water, a fixed sample mixed with an epoxy bond, and a cellular sample. In the cellular environment, the rotations of particles and magnetic moments were inhibited by particle-cell and dipole-dipole interactions, respectively. The heat dissipation of the MNPs in the cellular environment was lower than that of the liquid and fixed samples. Moreover, the SLP in a single cell was estimated. The temperature increase of a single cell was calculated on the basis of the conventional theoretical model and the SLP measured in a single cell.

\section{Introduction}

Hyperthermia induced by magnetic nanoparticles (MNPs) is a potent tool in cancer therapy [1-3]. To increase the therapeutic effect of magnetic hyperthermia, combination therapy with other clinical agents and evaluation of the method to synthesize MNPs have been studied. In particular, antibody-modified MNPs exhibited a therapeutic effect on cancer owing to their enhanced selectivity toward cancer cells $[4,5]$. The various sizes of spinal-structured iron oxide particles have been synthesized by changing heating time of $\alpha-\mathrm{Fe}_{2} \mathrm{O}_{3}$ platelet particles in tetraethylene glycol [6]. In addition to hyperthermia, magnetic particle imaging (MPI) has recently attracted attention as the application to visualize MNPs in the human body for therapy and diagnostics $[3,7]$.

The volumetric power dissipation $P$, as calculated according to the following equation, is used to quantify the heat generated by MNPs [8]:

$$
P=-f \mu_{0} \oint M d H=\mu_{0} \pi \chi^{\prime \prime} f H^{2},
$$

where $f, \mu_{0}, M, H$, and $\chi^{\prime \prime}$ are the frequency of magnetic field and the permeability of free space, the magnetization of MNPs, the magnetic field strength, and out-of-phase component of susceptibility, respectively. In addition, the specific and intrinsic loss powers-SLP and ILP-are used to express the mass power dissipation and the intrinsic heat dissipation, independent of the applied magnetic field and frequency $[9,10]$ :

$$
\begin{aligned}
& \text { SLP }=\frac{P}{\rho}, \\
& \text { ILP }=\frac{\text { SLP }}{f H^{2}},
\end{aligned}
$$

where $\rho$ is the mass density of the MNPs. The magnetic properties of MNPs in tumor tissue and in cellular environments must be measured to evaluate the heating capability of MNPs under therapeutic conditions. The SLP in cellular and intratumor conditions has been estimated by a calorimetric measurement [11-14]. The SLP has been shown to decrease in tumor tissue and in the cellular environments as a consequence of the inhibition of particle rotation due to particle-particle and particle-cellular tissue interactions [13, 14]. Immobilization in tumors and cells inhibited Brownian 


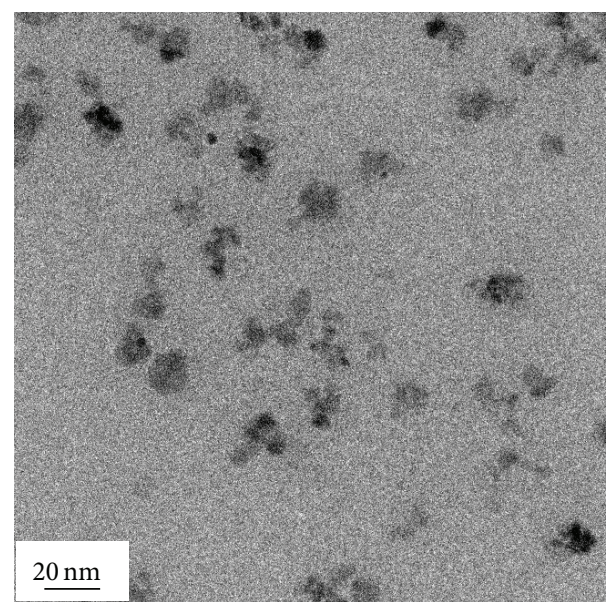

Figure 1: Transmission electron microscopic (TEM) image of the water-based magnetite nanoparticles.

relaxation. Moreover, a theoretical model for estimating the temperature increase of intratumor MNPs has been developed [15-17].

Magnetic relaxation loss is one of the heating mechanisms of MNPs, which is divided into Brownian and Néel relaxation. Brownian and Néel relaxation are associated with the rotation of particles and magnetic moments, respectively. Brownian and Néel relaxation times are obtained by the following equations [8]:

$$
\begin{aligned}
\tau_{\mathrm{B}} & =\frac{3 \eta V_{\mathrm{H}}}{k_{\mathrm{B}} T}, \\
\tau_{\mathrm{N}} & =\frac{\sqrt{\pi}}{2} \tau_{0} \frac{\exp \left(K V_{\mathrm{M}} / k_{\mathrm{B}} T\right)}{\sqrt{K V_{\mathrm{M}} / k_{\mathrm{B}} T}},
\end{aligned}
$$

where $\eta, V_{\mathrm{H}}, k_{\mathrm{B}}, T, \tau_{0}, K$, and $V_{\mathrm{M}}$ are the viscosity of the suspended fluid, the hydrodynamic volume of MNPs, the Boltzmann constant $1.38 \times 10^{-23} \mathrm{~J} / \mathrm{K}$, the temperature in Kelvin, the attempt time of $\sim 10^{-9} \mathrm{~s}$, the magnetocrystalline anisotropy constant, and the volume of the primary particle, respectively.

In this study, MNPs were dispersed in water, fixed with an epoxy bond, and added to cells. DC and AC hysteresis loops of each sample were measured. Magnetization reversal was evaluated on the basis of the AC hysteresis loops. Heat dissipation, as measured from the AC hysteresis loops, was not affected by a change in the state of the surrounding environment. The SLP and ILP were estimated from the area of the AC hysteresis loops. Moreover, the SLP in a single cell was calculated on the basis of the SLP of a cellular sample.

\section{Materials and Methods}

2.1. Materials. Water-based magnetite nanoparticles, Nanomag-D-spio, purchased from Micromod Partikeltechnologie GmbH, Rostock, Germany, were used in this study. The particle surface was functionalized by carboxylic acid group. Figure 1 shows the transmission electron microscopy (TEM) image of the nanoparticles. The shape and size were not so uniform. The primary diameter was varied from 4 to $14 \mathrm{~nm}$. The average was $9.8 \mathrm{~nm}$ with standard deviation of $2.0 \mathrm{~nm}$. Their hydrodynamic diameter measured by dynamic light scattering (DLS) was $40 \pm 16 \mathrm{~nm}$ [18].

2.2. Preparation of Liquid and Fixed Samples. A liquid sample consisting of MNPs dispersed in water was prepared. For the preparation of the fixed sample, MNPs dispersed in water were mixed with an epoxy bond. The concentration of MNPs in both the liquid and fixed samples was $2.8 \mathrm{mg}-\mathrm{Fe} / \mathrm{mL}$.

2.3. Preparation of Cellular Sample. HeLa cells (human cervical carcinoma line) were cultured in Dulbecco's modified Eagle medium (DMEM) supplemented with $10 \%$ fetal bovine serum (FBS) and $1 \%$ penicillin-streptomycin (PS) at $37^{\circ} \mathrm{C}$. HeLa cells were cultured in $100 \mathrm{~mm}$ dishes at a density of $9 \times$ $10^{5}$ cells/well. After $50 \mathrm{~h}$ of incubation, a MNP solution with a concentration of $12 \mu \mathrm{g}-\mathrm{Fe} / \mathrm{mL}$ was added to cells in $10 \mathrm{~mL}$ of serum-free culture medium and the cells were allowed to stand for $16 \mathrm{~h}$. After the cells were washed with phosphatebuffered saline (PBS), the cells were detached using 0.05\% trypsin EDTA and purified by centrifugation at 1,000 rpm for $5 \mathrm{~min}$. MNP-loaded cells were collected in a $2-\mathrm{mL}$ tube as a cellular sample. The final concentration of MNPs was $2.8 \mathrm{mg}-$ $\mathrm{Fe} / \mathrm{mL}$. The number of cells in the cellular sample was $7 \times$ $10^{7}$ cells. The biocompatibility of magnetite nanoparticles to HeLa cells was previously studied [19]. The viability of HeLa cells was above $80 \%$ for $48 \mathrm{~h}$ after adding the nanoparticles at the concentration of $72 \mu \mathrm{g}-\mathrm{Fe} / \mathrm{mL}$, which was higher than that used in this study. Figure 2 shows the images of detached HeLa cells measured as a cellular sample. Agglomerated MNPs were observed in the cytoplasm and on the membrane. The outer line of a cell indicates the membrane. The part inside the outer line of a cell was the cytoplasm because the outer line of the nucleus was clearly confirmed. Thus, MNPs observed in or around the outer line of a cell were evaluated as the intracellular or extracellular MNPs on the same plane, respectively. Although MNPs were internalized in cytoplasm, MNPs partially absorbed on the membrane were also confirmed. The diameters of MNP aggregations were measured and the volumes of MNP aggregations were calculated by the spherical approximation. The volume ratio of intracellular MNPs compared to MNPs both in the cytoplasm and on the membrane was approximately $81 \%$. It was also confirmed that the particle localizations were changed in the different focused plane. It has been reported that iron oxide nanoparticles are degraded by lysosome [20]. However, degradation of magnetite nanoparticles in the cellular sample was evaluated by measuring DC and AC hysteresis loops in this study. Magnetization of the particles was not affected, which indicated that degradation of the nanoparticles during a limited period of the experiment was negligible.

2.4. Measurement of DC and AC Hysteresis Loops. DC hysteresis loops were measured by vibrating sample magnetometry (VSM) using a DC magnetic field of 4 and $8 \mathrm{kA} / \mathrm{m}$ as minor loops and $0.8 \mathrm{MA} / \mathrm{m}$ as major loops. AC hysteresis loops were measured in the frequency range of $100-500 \mathrm{kHz}$ 


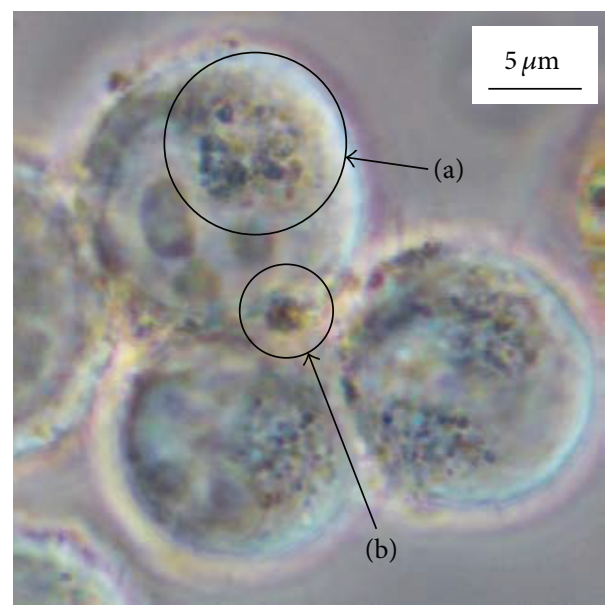

(a) Intracellular MNPs

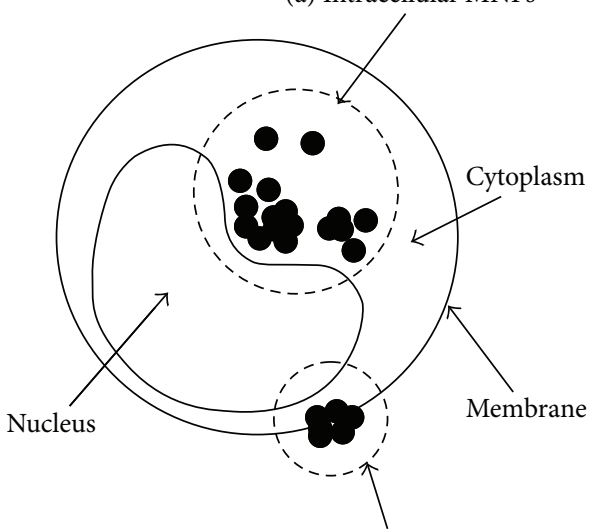

(b) Extracellular MNPs

FIGURE 2: Detached HeLa cells as a cellular sample. (a) Intracellular and (b) extracellular MNPs were observed.

under an AC magnetic field of 4 and $8 \mathrm{kA} / \mathrm{m}$ as minor loops. The exciting coil was a 70 -turn water-cooled solenoid with a $16.3 \mathrm{~mm}$ diameter [18]. All measurements were conducted at room temperature.

\section{Results and Discussion}

3.1. DC Hysteresis Loops. Figure 3 shows the DC major hysteresis loops of liquid, fixed, and cellular samples. Figure 3(b) shows the magnified images of Figure 3(a) at low magnetic field, which indicates the magnetization process in all of the samples. These samples exhibited superparamagnetism because the coercivity of the major loops in these samples was substantially low (Figure 3(b)). Anisotropic energy is given as the following equation:

$$
E(\theta)=K V_{\mathrm{M}} \sin ^{2}(\theta),
$$

where $\theta$ is the angle between the easy axis and magnetic moment. The anisotropy energy is low in small $\theta$. The particle rotation reduces anisotropy energy because the easy axis follows the direction of a magnetic moment (see (6)). The magnetization in the liquid sample was higher than that in the fixed sample during the magnetization reversal process (Figure 3(b)). The particles in the liquid sample are rotatable as opposed to the particles in the fixed sample. With respect to the liquid sample, the anisotropy energy barrier is decreased because particle rotation aligns the easy axis to the direction of the magnetic moment [21]. Therefore, the magnetic moment in the liquid sample was more rotatable than that in the fixed sample due to the lower anisotropy energy. For the superparamagnetic regime, magnetization reversal occurred due to the rotation of a magnetic moment whereas the reversal of a magnetic moment was associated with the magnetization reversal for the ferromagnetic regime. Although the magnetization in the liquid sample was higher than that in the fixed sample, the difference in the magnetization between the liquid and fixed samples was not significant. It is indicated that the effect of the particle rotation on the reduction of anisotropy energy is small because the random oscillation by the thermal energy is significantly influenced on the magnetization reversal for the superparamagnetic regime [22]. Thus, particle rotation slightly promoted the magnetization reversal in the liquid sample in comparison to that in the fixed sample. The magnetization in the cellular sample was lower than that in the fixed sample. The intracellular MNPs were encapsulated in endosomes and agglomerated in cytoplasm [23]. The extracellular MNPs were absorbed to the membrane in agglomerated state. In contrast to the cellular sample, MNPs were homogeneously dispersed in the fixed sample. The concentration of MNPs in the cellular sample was partially higher than that in the fixed sample because of the inhomogeneous agglomeration in the sample [24]. The interparticle distance is small at the higher concentration due to the inhomogeneous agglomeration. In the case of the smaller interparticle distance, the magnetization was decreased because the rotation of the magnetic moment was inhibited by dipole-dipole interaction [25]. Figure 4 shows the DC minor hysteresis loops of the liquid, fixed, and cellular samples. The minor loops exhibited no coercivity because of superparamagnetism. The magnetization of the fixed sample was lower than that of the liquid sample. In the liquid sample, the magnetic moments were reversed by the rotation of both the magnetic moments themselves and the particles. The reversal of the magnetic moment in the fixed sample was slower than that in the liquid sample because of the immobilized state in the fixed sample [13]. The magnetization of the cellular sample was lower than that of the fixed sample. Magnetization of the cellular sample was reduced as a consequence of dipole-dipole interaction due to inhomogeneous aggregation of MNPs in the cytoplasm and on the membrane [25].

3.2. AC Hysteresis Loops. Figure 5 shows AC hysteresis loops of the liquid, fixed, and cellular samples. The magnetization of the cellular sample was lower than that of the fixed sample, which was lower than that of the liquid sample. The magnetization of the AC minor hysteresis loops also indicates the magnetization reversal in the applied magnetic field range. With respect to the difference of the magnetization among all of the samples, the AC hysteresis loops showed the same trend with the DC minor hysteresis loops (Figures 3 and 4). The magnetization in the liquid sample was higher than that 


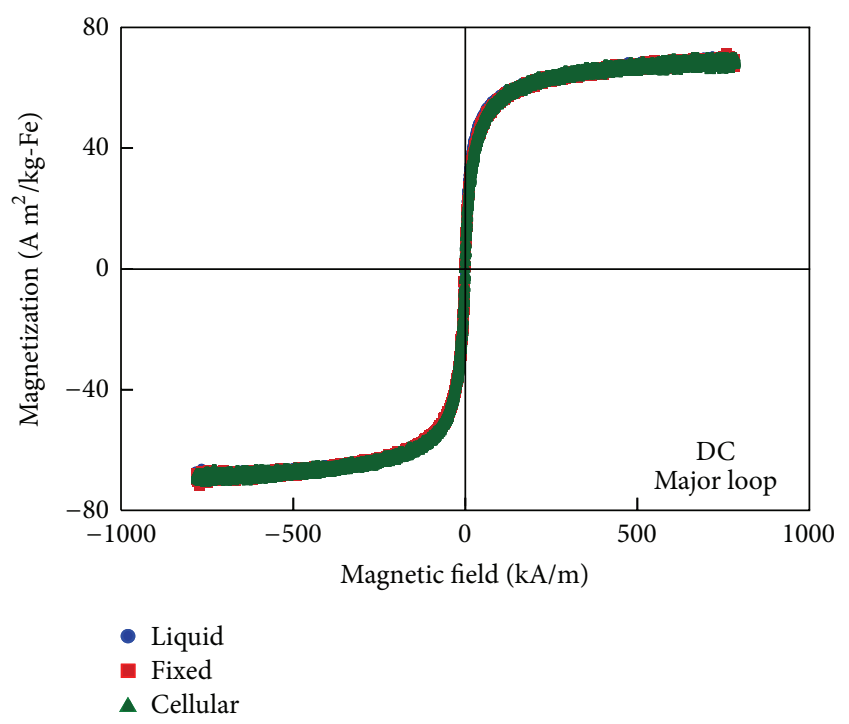

(a)

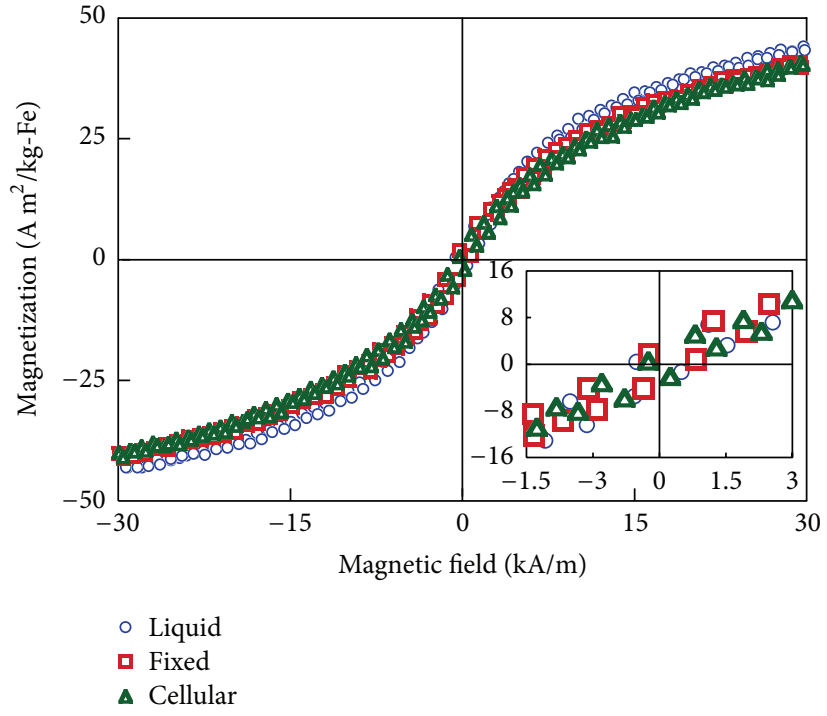

(b)

Figure 3: (a) DC major hysteresis loops of liquid, fixed, and cellular samples. The amplitude of the DC magnetic field was $0.8 \mathrm{MA} / \mathrm{m}$. (b) Magnified images of (a) at low magnetic field.

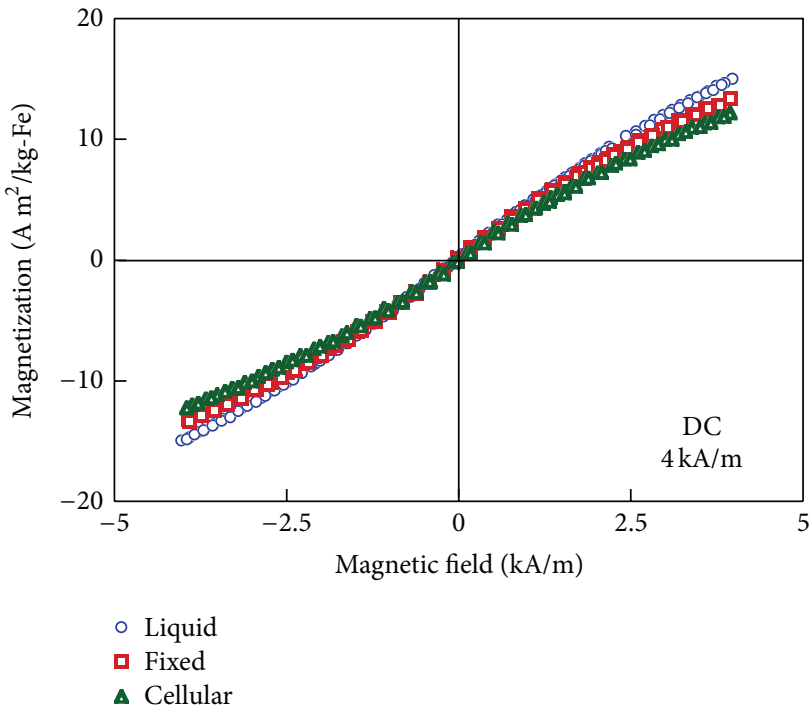

(a)

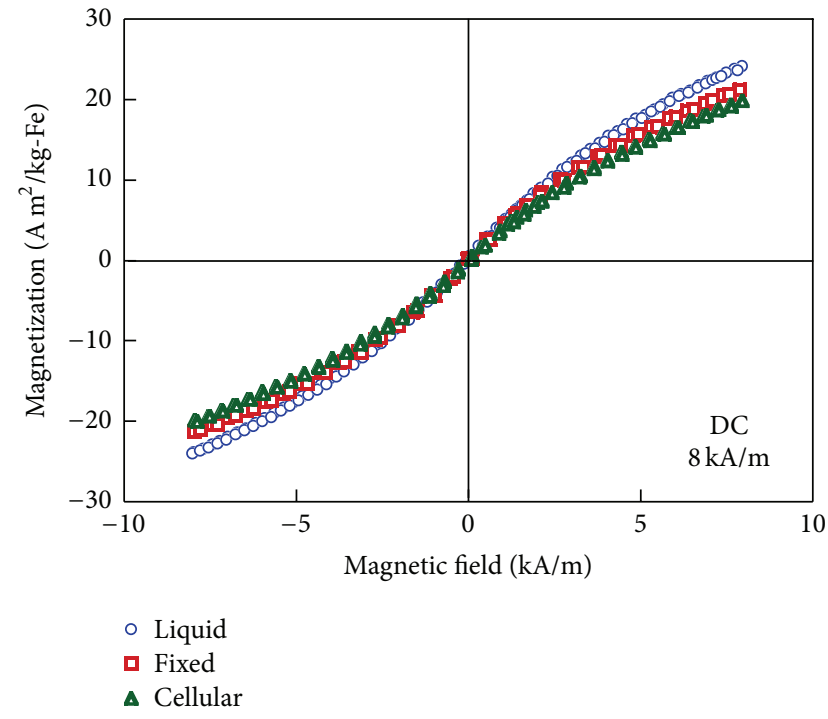

(b)

Figure 4: DC minor hysteresis loops of liquid, fixed, and cellular samples. The amplitude of the DC magnetic field was (a) $4 \mathrm{kA} / \mathrm{m}$ or (b) $8 \mathrm{kA} / \mathrm{m}$.

in the fixed sample because of the anisotropy energy reduced by the particle rotation. In the cellular sample, the rotation of magnetic moments was inhibited due to dipole-dipole interaction induced by the inhomogeneous aggregation in the cellular environment. Unlike the DC minor hysteresis loops, the AC minor hysteresis loops revealed hysteresis areas in all of the samples because the rotation of the magnetic moments was delayed compared to the applied magnetic field. The results indicated that magnetic relaxation occurred due to a delay in the rotation of the magnetic moment in all of the samples. As indicated by the hysteresis loops of the fixed sample in which the particles were not rotatable, Néel relaxation occurred in all of the samples, which exhibited areas. The coercivity of the liquid sample was higher than that of the fixed sample. Thus, Brownian relaxation occurred only in the liquid sample because the particles in this sample were rotatable [26]. Moreover, the coercivity of the cellular sample was the same as that of the fixed sample. It was indicated that delay of the rotation in magnetic moments in the cellular sample was similar to the fixed sample. These results indicate that particle rotation was inhibited by particle-cell interactions in the cellular environment. Only Néel relaxation occurred in the cellular sample $[11,13,14]$. 


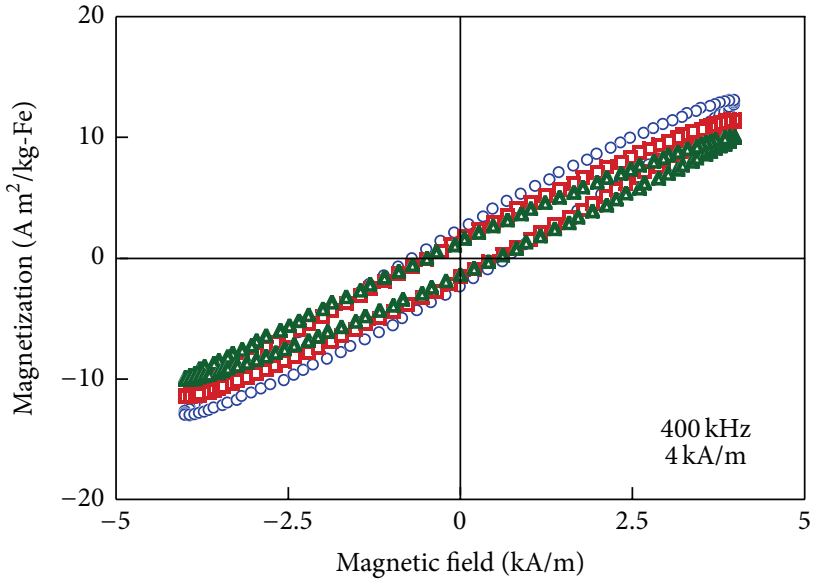

(a)

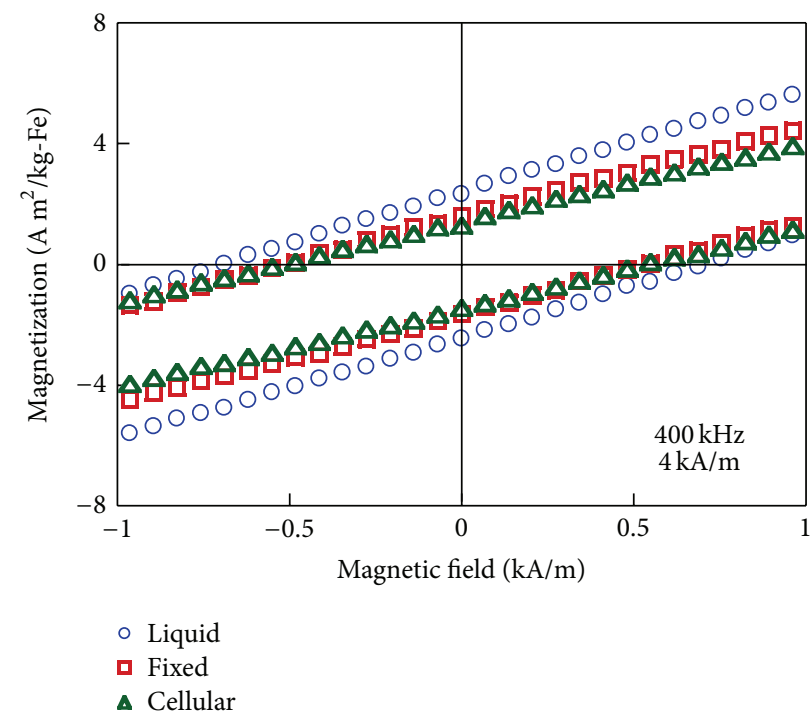

(c)

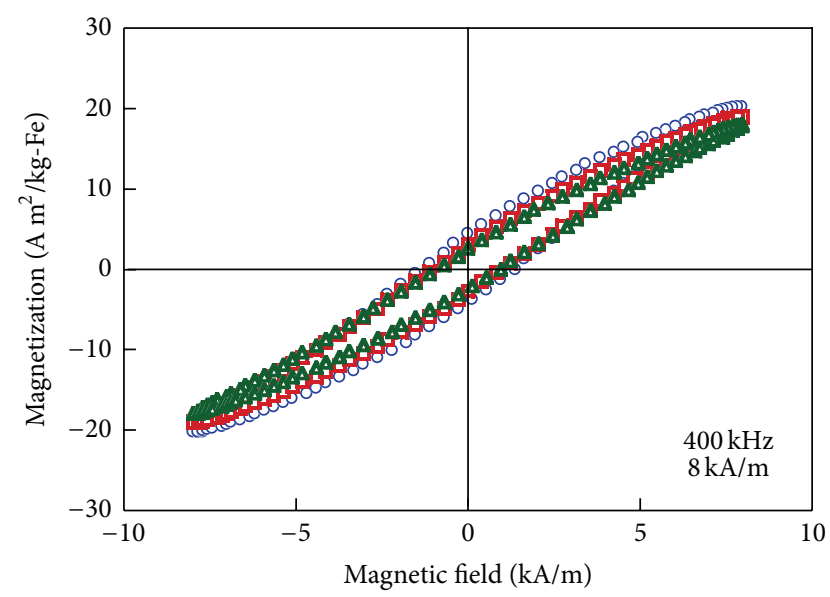

(b)

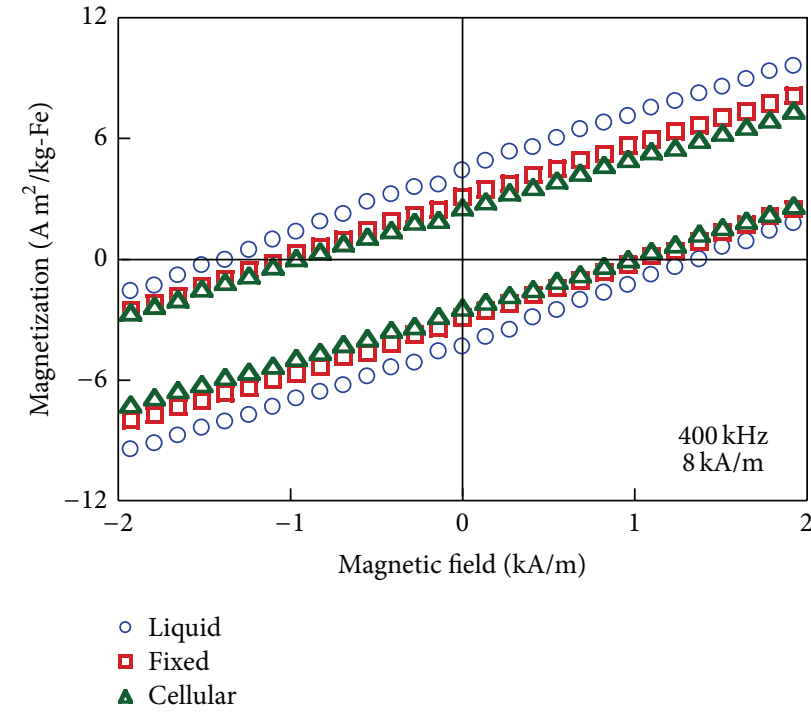

(d)

FIGURE 5: AC minor hysteresis loops of liquid, fixed, and cellular samples. The amplitude of the AC magnetic field was (a, c) $4 \mathrm{kA} / \mathrm{m}$ or (b, d) $8 \mathrm{kA} / \mathrm{m}$. The frequency was $400 \mathrm{kHz}$. Magnified images of (a) and (b) are shown in (c) and (d), respectively.

3.3. Heat Dissipation Estimated from AC Hysteresis Loops. The SLP was estimated from the area of the AC hysteresis loops. The ILP was calculated from the measured SLP and from (3). Figures 6 and 7, respectively, show the dependence of the SLP and ILP on the frequency of an applied magnetic field. By (5), the theoretical frequency of Néel relaxation peak $f_{N}$ is higher than $2.2 \mathrm{MHz}\left(K \leq 41 \mathrm{~kJ} / \mathrm{m}^{3}\right)[8,27,28]$. The measured frequency range in this study was lower than $f_{N}$. With increasing frequency, the ILP increased, the heat dissipation derived from the magnetic relaxation loss, and the rotation of the magnetic moments was gradually delayed. The decrease of the ILP between the liquid and fixed samples is associated with the inhibition of Brownian relaxation in the fixed sample. By (4), the theoretical frequency of Brownian relaxation peak $f_{B}$ is $7.3 \mathrm{kHz}\left(\eta=\eta_{\text {water }}=0.89 \mathrm{mPa} \cdot \mathrm{s}\right)$. The theoretical $f_{B}$ was lower than the measured frequency range, $100-500 \mathrm{kHz}$. However, higher coercivity in the liquid sample than in the fixed sample shows occurrence of Brownian relaxation due to delay of particle rotation in the measured frequency range. The SLP of the intracellular MNPs has been estimated by a calorimetric measurement [11]. It has been reported that the SLP of intracellular MNPs was lower than that of MNPs dispersed in water because the Brownian relaxation was abrogated. With respect to the reduction of heat dissipation by the inhibition of Brownian relaxation, this study showed the same result with the conventional study. However, in this study, the measurements of magnetization reversal by DC and AC hysteresis loops also revealed that heat dissipation of MNPs in the cellular environment was lower than that of fixed MNPs because of the lower magnetization (Figures 4 and 5). The ILP of the cellular sample was lower than that of the fixed sample, as indicated by the lower magnetization of the cellular sample due to dipoledipole interaction. The SLP is proportional to $H^{2}$ when magnetization is linear with magnetic field (see (1) and (2)). However, magnetization was not linear with magnetic field 


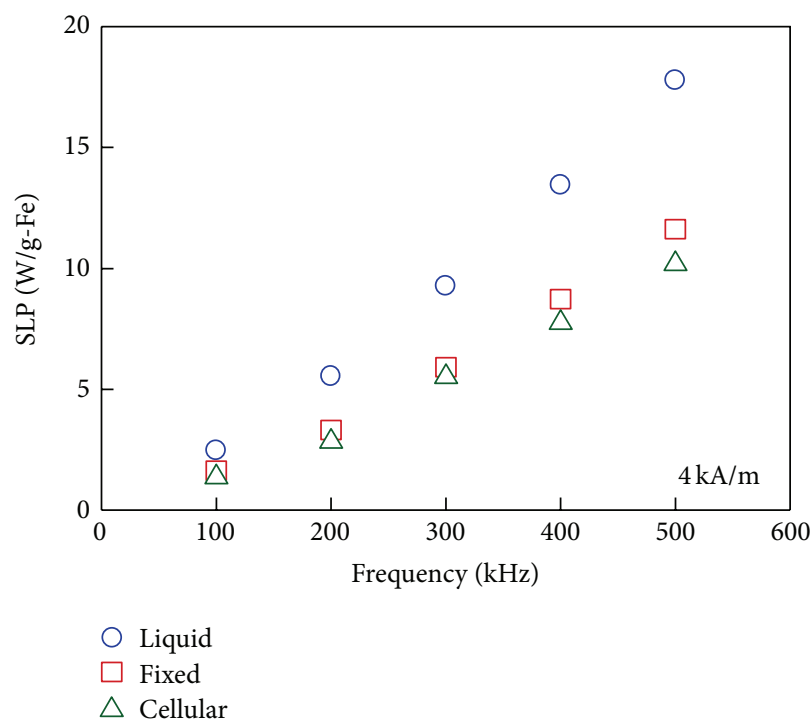

(a)

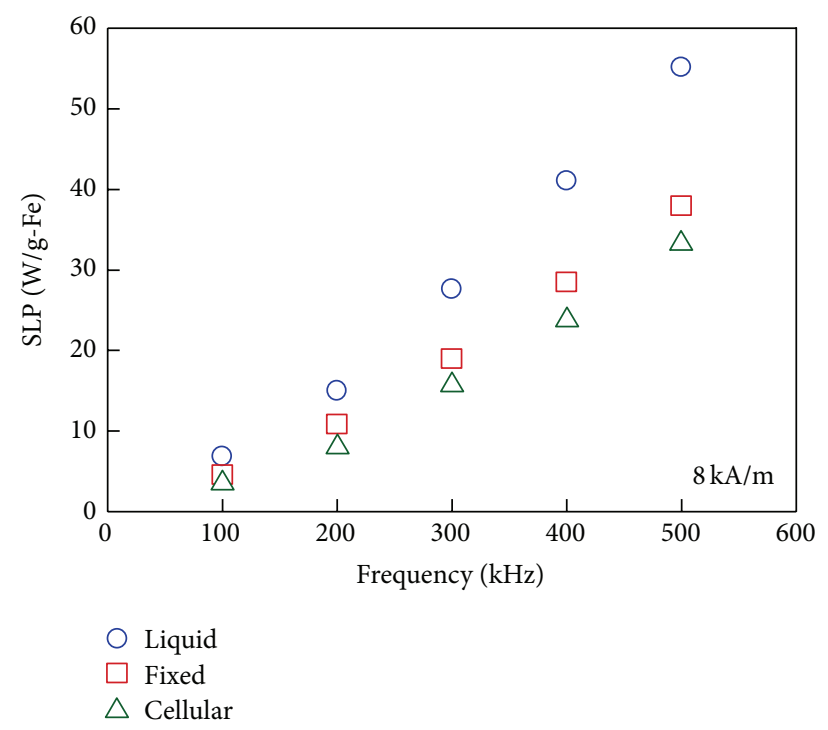

(b)

FIGURE 6: The dependence of SLP estimated from the areas of AC hysteresis loops on frequency. The amplitude of the magnetic field was (a) $4 \mathrm{kA} / \mathrm{m}$ or (b) $8 \mathrm{kA} / \mathrm{m}$.

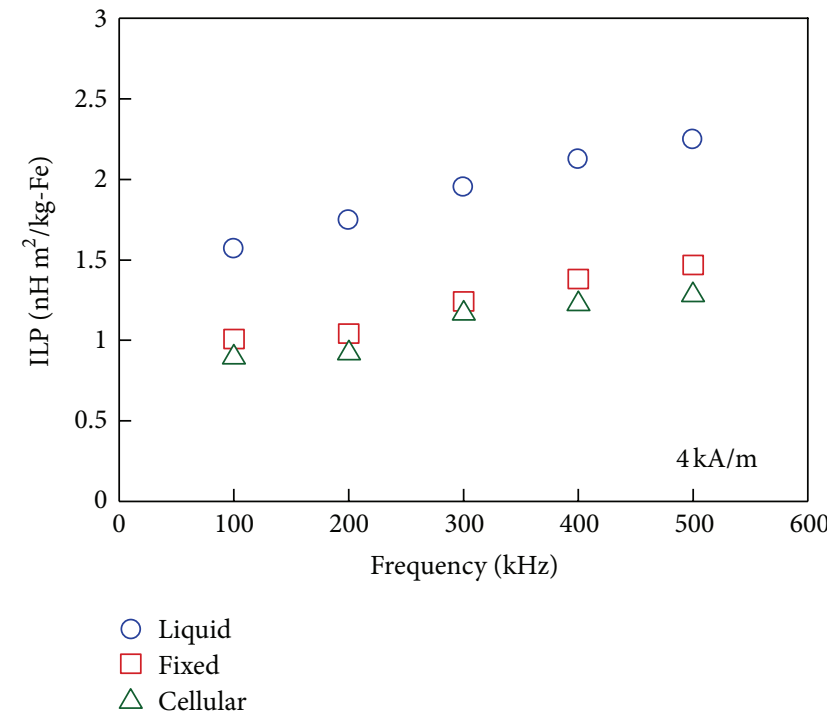

(a)

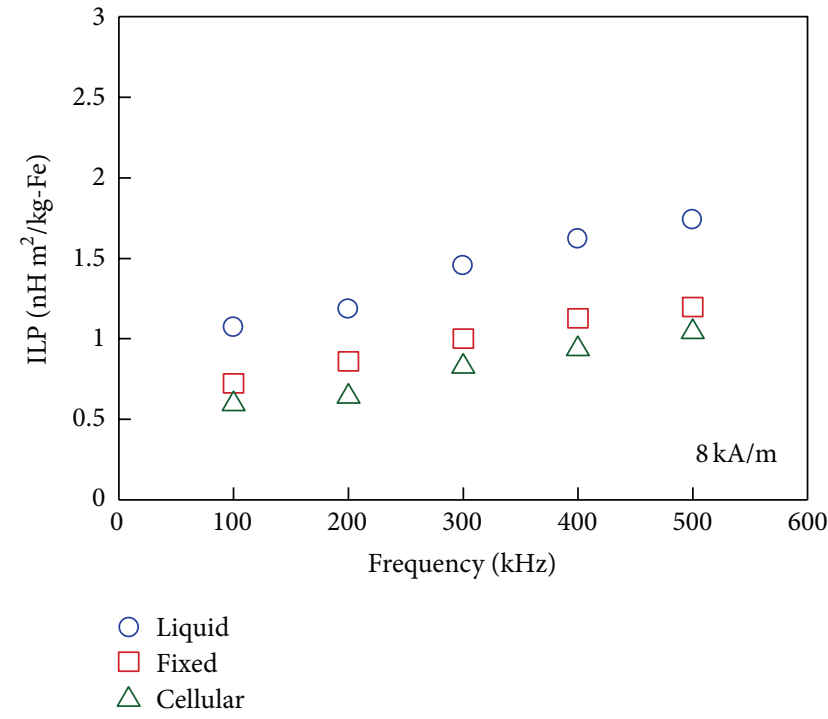

(b)

FIGURE 7: The dependence of the ILP calculated from the measured SLP (Figure 6) and (3) on frequency. The amplitude of the magnetic field was (a) $4 \mathrm{kA} / \mathrm{m}$ or (b) $8 \mathrm{kA} / \mathrm{m}$.

in applied magnetic field range. The ILP at $8 \mathrm{kA} / \mathrm{m}$ was lower than that at $4 \mathrm{kA} / \mathrm{m}$. In the case of SLP being unproportional to $H^{2}$, the ILP is lower at the higher magnetic field because the ILP is shown as the SLP normalized by $H^{2} f$ (see (3)).

The SLP in a single cell was estimated (Figure 8 ) using the following equation:

$$
\operatorname{SLP}_{\text {single cell }}=\operatorname{SLP}_{\text {cellular }} \frac{m_{\mathrm{Fe}}}{N_{\text {cell }}}
$$

where SLP $\mathrm{Cellular}_{\mathrm{Fe}}, m_{\mathrm{Fe}}$, and $N_{\text {cell }}$ are the SLP, the mass of Fe, and the number of cells in the cellular sample, respectively.
The temperature increase at the center of a heated spherical object is estimated from the following equation [15-17]:

$$
\Delta T_{\mathrm{s}}=\frac{D P}{8 k_{\mathrm{s}}},
$$

where $D, P$, and $k_{\mathrm{s}}$ are the diameter of the sphere, the heating rate per unit volume within the sphere, and the thermal conductivity of the sphere, respectively. Applying the spherical model in (8) to a single cell, (8) was obtained as

$$
\Delta T_{\text {single cell }}=\frac{D_{\text {cell }}}{8 k_{\text {cell }}} \frac{\operatorname{SLP}_{\text {single cell }}}{V_{\text {cell }}},
$$




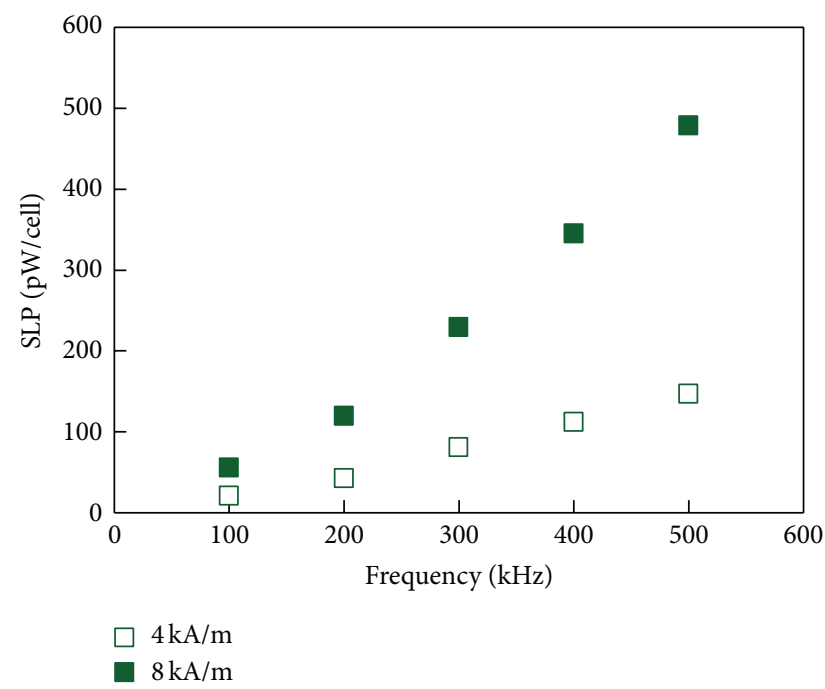

FIGURE 8: The dependence of the SLP in a single cell on frequency, as calculated from (7).

where $D_{\text {cell }}, V_{\text {cell }}$, and $k_{\text {cell }}$ are the diameter and volume of a single cell and the thermal conductivity of a cell $\left(0.64 \mathrm{~W} / \mathrm{m}^{\circ} \mathrm{C}\right)$, respectively. In Figure 8 , the SLP in a single cell was $480 \mathrm{pW} /$ cell at $8 \mathrm{kA} / \mathrm{m}$ and $500 \mathrm{kHz}$. In the case of $D_{\text {cell }}=15 \mu \mathrm{m}$, the temperature increase of a single cell was 12 $\times 10^{-6 \circ} \mathrm{C}$. Therefore, at $8 \mathrm{kA} / \mathrm{m}$ and $500 \mathrm{kHz}$, the SLP of MNPs necessary to kill a single cell by hyperthermia treatment $\left(\Delta T=5^{\circ} \mathrm{C}\right)$ was $14 \mathrm{MW} / \mathrm{g}$-Fe because the SLP of the cellular sample was $33.6 \mathrm{~W} / \mathrm{g}-\mathrm{Fe}$. This result is in good agreement with the conventional estimation of SLP necessary to kill a single cell [29]. It was confirmed that hyperthermia focused on a single cell was restricted due to the limitation in the amount of MNPs internalized into a single cell $[30,31]$.

\section{Conclusion}

The heating mechanism and heat dissipation of MNPs in the cellular environment were assessed via measurements of DC and AC hysteresis loops. In the intracellular state, the rotations of particles and magnetic moments were inhibited due to particle-cell and dipole-dipole interactions, respectively. Therefore, the heat dissipation of MNPs in the cellular environment was lower than that of MNPs in the liquid and fixed states, because Brownian relaxation was inhibited and magnetization decreased in a cell. It was also revealed that heat dissipation of MNPs in the cellular environment was reduced by dipole-dipole interaction due to the partial agglomeration of MNPs. Our results indicated that because the concentration of MNPs in the cells was low, the hyperthermia treatment was not conducted solely by intracellular hyperthermia. High intratumoral accumulation of MNPs in both intra- and extracellular states is necessary to induce hyperthermia.

\section{Conflict of Interests}

The authors declare that there is no conflict of interests regarding the publication of this paper.

\section{References}

[1] C. C. Berry and A. S. G. Curtis, "Functionalisation of magnetic nanoparticles for applications in biomedicine," Journal of Physics D: Applied Physics, vol. 36, no. 13, pp. R198-R206, 2003.

[2] Q. A. Pankhurst, N. K. T. Thanh, S. K. Jones, and J. Dobson, "Progress in applications of magnetic nanoparticles in biomedicine," Journal of Physics D: Applied Physics, vol. 42, Article ID 224001, 2009.

[3] K. M. Krishnan, "Biomedical nanomagnetics: a spin through possibilities in imaging, diagnostics, and therapy," IEEE Transactions on Magnetics, vol. 46, no. 7, pp. 2523-2558, 2010.

[4] A. Ito, K. Tanaka, K. Kondo et al., "Tumor regression by combined immunotherapy and hyperthermia using magnetic nanoparticles in an experimental subcutaneous murine melanoma," Cancer Science, vol. 94, no. 3, pp. 308-313, 2003.

[5] S. Ota, N. Yamazaki, A. Tomitaka, T. Yamada, and Y. Takemura, "Hyperthermia using antibody-conjugated magnetic nanoparticles and its enhanced effect with cryptotanshinone," Nanomaterials, vol. 4, no. 2, pp. 319-330, 2014.

[6] M. Kishimoto, K. Asai, A. Horiuchi, H. Yanagihara, Y. Nagasaki, and E. Kita, "Characterization of spinel-structured iron oxide particles synthesized by heating $\alpha-\mathrm{Fe}_{2} \mathrm{O}_{3}$ platelets in tetraethylene glycol," Materials Transactions, vol. 54, no. 2, pp. 222224, 2013.

[7] B. Gleich and J. Weizenecker, "Tomographic imaging using the nonlinear response of magnetic particles," Nature, vol. 435, no. 7046, pp. 1214-1217, 2005.

[8] R. E. Rosensweig, "Heating magnetic fluid with alternating magnetic field," Journal of Magnetism and Magnetic Materials, vol. 252, no. 1-3, pp. 370-374, 2002.

[9] S. Dutz and R. Hergt, "Magnetic nanoparticle heating and heat transfer on a microscale: basic principles, realities and physical limitations of hyperthermia for tumour therapy," International Journal of Hyperthermia, vol. 29, no. 8, pp. 790-800, 2013.

[10] M. Kallumadil, M. Tada, T. Nakagawa, M. Abe, P. Southern, and Q. A. Pankhurst, "Suitability of commercial colloids for magnetic hyperthermia," Journal of Magnetism and Magnetic Materials, vol. 321, no. 10, pp. 1509-1513, 2009.

[11] J.-P. Fortin, F. Gazeau, and C. Wilhelm, "Intracellular heating of living cells through Néel relaxation of magnetic nanoparticles," European Biophysics Journal, vol. 37, no. 2, pp. 223-228, 2008.

[12] C. L. Dennis, A. J. Jackson, J. A. Borchers et al., "Nearly complete regression of tumors via collective behavior of magnetic nanoparticles in hyperthermia," Nanotechnology, vol. 20, no. 39, Article ID 395103, 2009.

[13] S. Dutz, M. Kettering, I. Hilger, R. Müller, and M. Zeisberger, "Magnetic multicore nanoparticles for hyperthermia-influence of particle immobilization in tumour tissue on magnetic properties," Nanotechnology, vol. 22, no. 26, Article ID 265102, 2011.

[14] R. D. Corato, A. Espinosa, L. Lartigue et al., "Magnetic hyperthermia efficiency in the cellular environment fordifferent nanoparticle designs," Biomaterials, vol. 35, no. 24, pp. 64006411, 2014.

[15] W. Andrä, C. G. D’Ambly, R. Hergt, I. Hilger, and W. A. Kaiser, "Temperature distribution as function of time around a small spherical heat source of local magnetic hyperthermia," Journal of Magnetism and Magnetic Materials, vol. 194, no. 1, pp. 197203, 1999.

[16] Y. Rabin, "Is intracellular hyperthermia superior to extracellular hyperthermia in the thermal sense?" International Journal of Hyperthermia, vol. 18, no. 3, pp. 194-202, 2002. 
[17] M. Hedayati, O. Thomas, B. Abubaker-Sharif et al., "The effect of cell cluster size on intracellular nanoparticle-mediated hyperthermia: is it possible to treat microscopic tumors?" Nanomedicine, vol. 8, no. 1, pp. 29-41, 2013.

[18] K. Nakamura, K. Ueda, A. Tomitaka, T. Yamada, and Y. Takemura, "Self-heating temperature and AC hysteresis of magnetic iron oxide nanoparticles and their dependence on secondary particle size," IEEE Transactions on Magnetics, vol. 49, no. 1, pp. 240-243, 2013.

[19] A. Tomitaka, A. Hirukawa, T. Yamada, S. Morishita, and Y. Takemura, "Biocompatibility of various ferrite nanoparticles evaluated by in vitro cytotoxicity assays using HeLa cells," Journal of Magnetism and Magnetic Materials, vol. 321, no. 10, pp. 1482-1484, 2009.

[20] K. Briley-Saebo, A. Bjørnerud, D. Grant, H. Ahlstrom, T. Berg, and G. M. Kindberg, "Hepatic cellular distribution and degradation of iron oxide nanoparticles following single intravenous injection in rats: implications for magnetic resonance imaging," Cell and Tissue Research, vol. 316, no. 3, pp. 315-323, 2004.

[21] J. Carrey, B. Mehdaoui, and M. Respaud, "Simple models for dynamic hysteresis loop calculations of magnetic singledomain nanoparticles: application to magnetic hyperthermia optimization," Journal of Applied Physics, vol. 109, Article ID 083921, pp. 1-17, 2011.

[22] D. Serantes, D. Baldomir, C. Martinez-Boubeta et al., "Influence of dipolar interactions on hyperthermia properties of ferromagnetic particles," Journal of Applied Physics, vol. 108, no. 7, Article ID 073918, 2010.

[23] C. Wilhelm, C. Billotey, J. Roger, J. N. Pons, J.-C. Bacri, and F. Gazeau, "Intracellular uptake of anionic superparamagnetic nanoparticles as a function of their surface coating," Biomaterials, vol. 24, no. 6, pp. 1001-1011, 2003.

[24] H. Mamiya and B. Jeyadevan, "Hyperthermic effects of dissipative structures of magnetic nanoparticles in large alternating magnetic fields," Scientific Reports, vol. 1, article 157, 2011.

[25] S. Dutz and R. Hergt, "The role of interactions in systems of single domain ferrimagnetic iron oxide nanoparticles," Journal of Nano- and Electronic Physics, vol. 4, no. 2, Article ID 02010, 2012.

[26] A. Tomitaka, K. Ueda, T. Yamada, and Y. Takemura, "Heat dissipation and magnetic properties of surface-coated $\mathrm{Fe}_{3} \mathrm{O}_{4}$ nanoparticles for biomedical applications," Journal of Magnetism and Magnetic Materials, vol. 324, no. 21, pp. 3437-3442, 2012.

[27] P. C. Fannin, C. N. Marin, and C. Couper, "Investigation of magnetic fluids exhibiting field-induced increasing loss peaks," Journal of Magnetism and Magnetic Materials, vol. 322, no. 9-12, pp. 1677-1681, 2010.

[28] M. Suto, Y. Hirota, H. Mamiya et al., "Heat dissipation mechanism of magnetite nanoparticles in magnetic fluid hyperthermia," Journal of Magnetism and Magnetic Materials, vol. 321, no. 10, pp. 1493-1496, 2009.

[29] R. Hergt and S. Dutz, "Magnetic particle hyperthermiabiophysical limitations of a visionary tumour therapy," Journal of Magnetism and Magnetic Materials, vol. 311, no. 1, pp. 187-192, 2007.

[30] C. Wilhelm, F. Gazeau, J. Roger, J. N. Pons, and J. C. Bacri, "Interaction of anionic superparamagnetic nanoparticles with cells: kinetic analyses of membrane adsorption and subsequent internalization," Langmuir, vol. 18, no. 21, pp. 8148-8155, 2002.
[31] C. Shundo, H. Zhang, T. Nakanishi, and T. Osaka, "Cytotoxicity evaluation of magnetite $\left(\mathrm{Fe}_{3} \mathrm{O}_{4}\right)$ nanoparticles in mouse embryonic stem cells," Colloids and Surfaces B: Biointerfaces, vol. 97, pp. 221-225, 2012. 

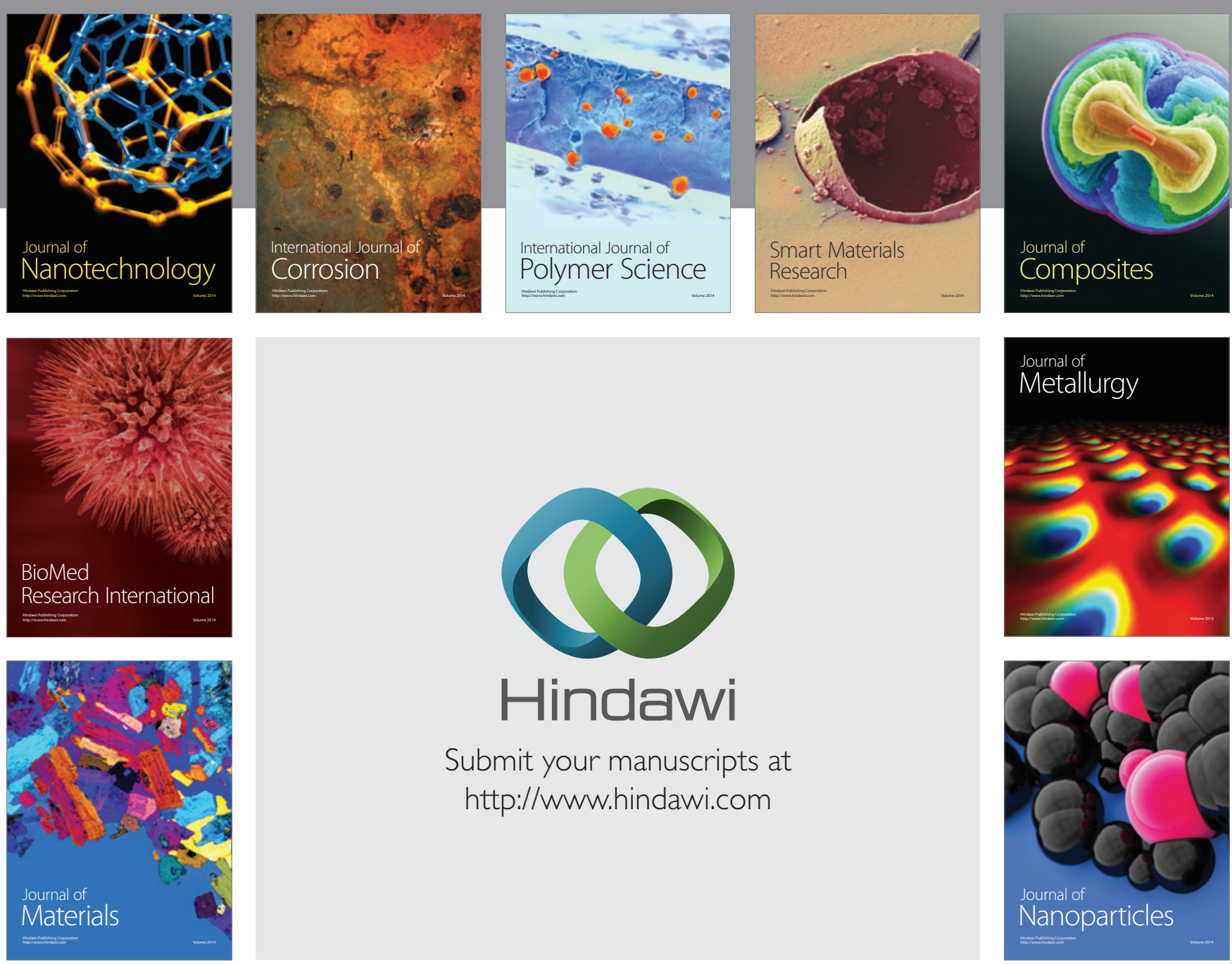

Submit your manuscripts at http://www.hindawi.com
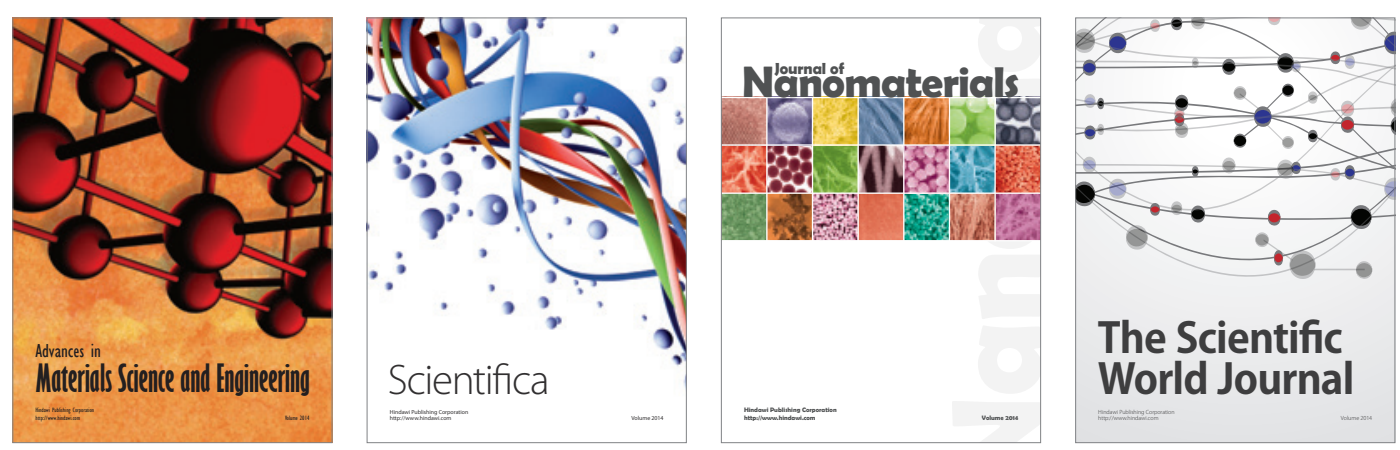

\section{The Scientific World Journal}
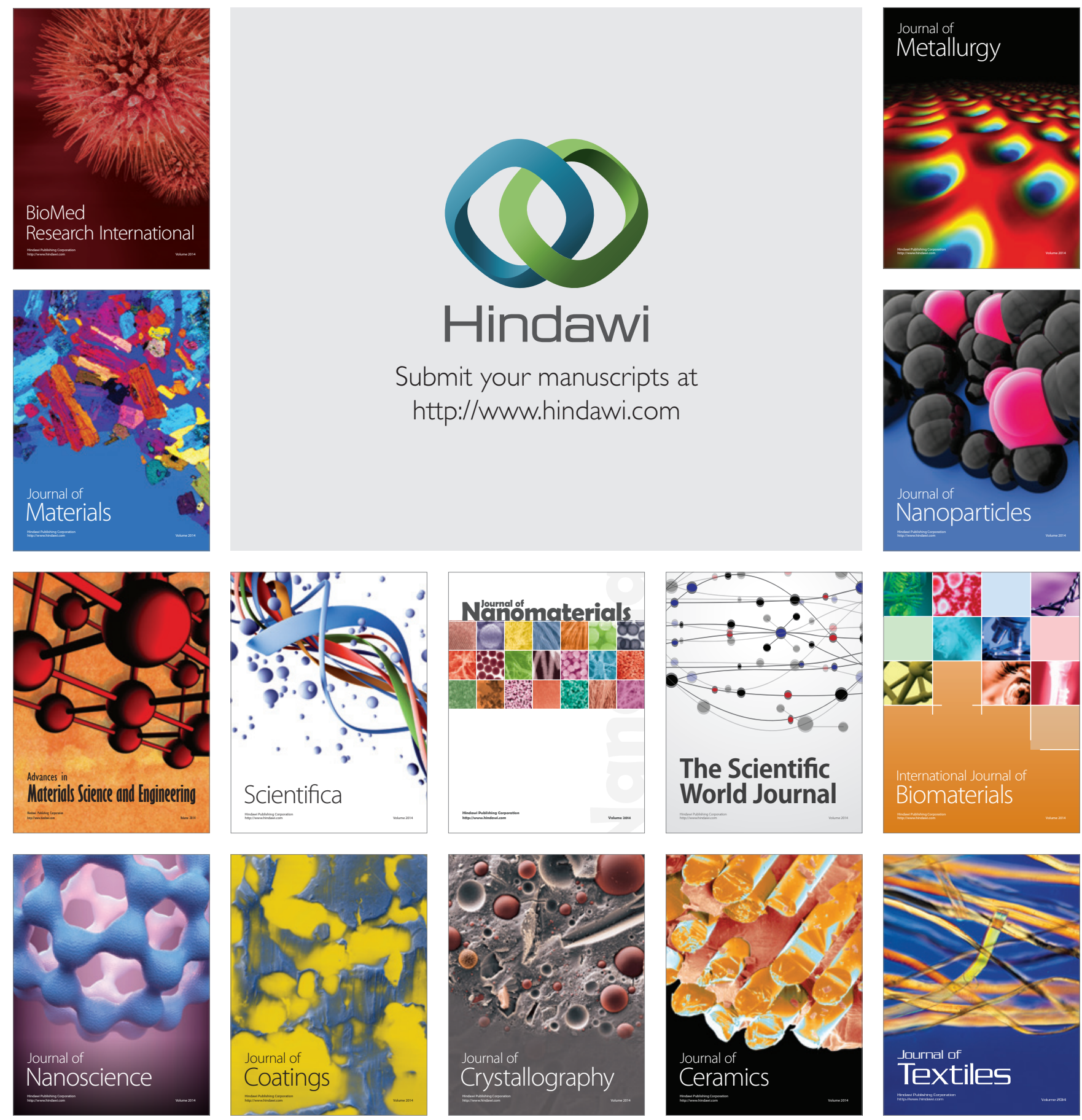